Journal of Economics and Behavioral Studies

Vol. 7, No. 3, pp. 14-22, June 2015 (ISSN: 2220-6140)

\title{
Validating and Identifying Health and Safety Performance Improvement Indicators: Experience of Using Delphi Technique
}

\author{
${ }^{1}$ Justus N. Agumba*, ${ }^{2}$ Theo Haupt \\ ${ }^{1}$ University of Johannesburg, South Africa \\ ${ }^{2}$ University of Kwazulu Natal, Durban, South Africa \\ *jagumba@uj.ac.za
}

\begin{abstract}
The literature on health and safety (H\&S) is bound with different elements and indicators of measuring H\&S performance. The purpose of this paper is to discuss the success and challenges of using quantitative and qualitative approach of Delphi technique in validating and identifying $\mathrm{H} \& \mathrm{~S}$ performance indicators that small and medium construction enterprises (SMEs) can use to measure and monitor their H\&S performance improvement at project level. Furthermore, discuss the identification of experts in the field of H\&S, ways of improving consensus analysis and minimizing experts' non-response. This study is based on practical experience of the researchers pertaining to the Delphi technique method which is a constructivist or interpretive approach to knowledge. The study started with an extensive literature review to identify core elements and leading indicators characterizing H\&S culture to develop a Delphi questionnaire that was used in the first round of Delphi. A four round Delphi technique was conducted to attain consensus of the identified $\mathrm{H} \& \mathrm{~S}$ indicators. The experts were identified from published articles of $\mathrm{H} \& S$, practitioners' website of H\&S and word of mouth. The use of email was used as a mode of communication. This study set to warn but also encourage the use of Delphi technique as a method to unearth information in areas where consensus has not been reached such as H\&S performance measurement indicators for construction SMEs in South Africa.
\end{abstract}

Keywords: Delphi technique, indicators; methodology, quantitative and qualitative

\section{Introduction}

The literature on health and safety culture is abound, with different measures, Fernandez et al. (2007) indicated a lack of consensus of the indicators that constitute health and safety management system which is a critical component of H\&S culture. Critical reading on H\&S indicators to be used for measuring performance improvement in H\&S has scantly focused on Delphi method to validate and identify the leading indicators i.e. management commitment and involvement in $\mathrm{H} \& S$, employee involvement and empowerment in $\mathrm{H} \& \mathrm{~S}, \mathrm{H} \& \mathrm{~S}$ resources, H\&S training, but to name a few. The few studies that have prompted the need for this study were undertaken in the United States of America. These were studies conducted by Hallowell (2008) and Rajendran (2007). Furthermore, Hallowell \& Gambatese (2010) indicated that Delphi technique can be used to finalize decisions when there is lack of empirical evidence that need to be made by experts. This statement supports the need to identify critical leading indicators for measuring H\&S performance using the Delphi method. Hence, the study was designed to use both quantitative and qualitative approach as the Delphi method straddles between these two approaches. It was to validated and identify, the H\&S leading indicators, based on opinions and judgment of H\&S experts in successive rounds of iteration that could be used to comprehensively articulate the indicators for H\&S performance improvement model for SMEs. It is worth noting that lagging indicators i.e. injuries, accidents and number of deaths have been used to measure H\&S performance. However, this measure is inadequate as it reports on the aftermath of what could have been prevented using leading indicators of H\&S. This article therefore focuses upon primary research phase of the project where quantitative and qualitative approach of collecting data was used in order to develop a final questionnaire to test the theory using structural equation modeling with construction SMEs. This was a significant methodology choice for the second phase of this research project. 


\section{Methodology}

It was obvious from the literature review that H\&S measures or indicators of H\&S culture are a controversial subject in the construction industry that continues to provoke debate i.e. there are not set H\&S indicators that are tied to H\&S culture, the indicators differ from study to study (see Fernandez-Muniz et al., 2007). A research method that was required that could generate and encourage the discussion of different opinions, in the attempt to ensure that all relevant issues were validated, identified and explored (Goldschmidt, 1996) was advocated for, this immediately ruled out a one-off questionnaire, which could elicit opinions but not encourage an exploration of these opinions. Constraint of time, cost and geography also ruled out a series of individual interviews or focus groups. The Delphi method was chosen for this second phase of the PhD research project. This method suited the requirements of this research study, as one of its characteristic is to provoke discussion and assist in reaching consensus on various indicators of $H \& S$ that will improve $H \& S$ performance of small and medium construction enterprise in South Africa, while also fitting the practical constraints of the duration of this research project.

Defining the Delphi Technique: Linstone and Turoff, (1975) refused to posit an explicit definition of Delphi method for two major reasons. Firstly, they believed that a research technique should be continuously evolving and redefining itself as it is applied to different areas of research, when something has attained a point at which it explicitly definable then progress has stopped. Most importantly they believed that in its design and use Delphi is more of an art than a science. Cape (2004) in his analysis further opines that the pioneers of Delphi method did not want to constrain the researcher by stating that Delphi should be used in one specific way, and only in certain areas of research. The researcher should be free to make the Delphi method their own, to tailor the technique to suit their own requirements. Nevertheless, general descriptions of the Delphi method must be provided before the researcher can adapt the technique to his/her own research. Delphi is usually used for collecting and distilling knowledge from experts (Ziglio, 1996). The researcher purposefully selects respondents with the knowledge and experience necessary to provide useful insight into the problem or issue under investigation. The experts are asked to answer a question or series of questions. This is usually done anonymously; the experts are in contact with the researcher but not with each other. The researcher analyses the views of the experts and returns them for further comment, again ensuring anonymity. This process is repeated over a set number of rounds, allowing the experts to alter or defend their views in the light of what others have said. A well designed Delphi survey should produce; explicit reasoned, self-aware opinions, expressed in the light of the opinions of associate experts (Dyer, 1979).

Methods of achieving high response rate: In line with the aforementioned discussion of Delphi, in order to improve the response rate of experts Hsu \& Sandford (2007) advocates for the following approaches;

Assistance from endorsed individuals: Hsu \& Sandford (2007) indicated that an expert endorsement or recommendation can help in identifying other experts. A list of expert panelists should be prepared, and there validity approved by an expert.

Initial contact: An initial contact has to be undertaken before the first round of Delphi is administered. The first contact is required where the approved experts are contacted telephonically an explanation of the research objectives is explained to them. If the identified experts are unwilling to participate they need to inform the researcher (Hsu and Sandford, 2007).

Open-ended vs. close-ended statements: According to Hsu and Sandford (2007) the use of a close-ended questionnaire with specific statements is viewed as an advantage than using open-ended questionnaire. From the view point of a participant, if a questionnaire is easy to respond to and less time-consuming, he/she is more likely to complete and return the questionnaire. Hsu and Sandford (2007) further indicates that the use of the open-ended questionnaire which is the traditional Delphi method is necessary if basic information regarding the target issue is unavailable

Dealing with non-respondents: Ludwig (1994) indicated that a drawback to Delphi method was the questionnaire method, which may slow the process greatly as several days or weeks may pass between rounds. Being an iterative method and sequential, the problem of how to accelerate the process of data collection poses a great challenge for Delphi researchers. The need for sending reminders using telephone contact or e-mail is recommended and setting deadlines in successive rounds is viewed to be important as Delphi method involves iteration (Hsu \& Sandford, 2007). 
Incentives: Providing incentives to help increase response rates is well documented in the literature (James \& Bolstein, 1992). Hsu \& Sandford (2007) indicate that researchers using Delphi method need to prepare incentives for different rounds. In addition to using incentives it is also beneficial for researchers to enclose thank-you note for the purpose of expressing gratitude for the panelists' responses and ongoing participation.

\section{Procedure of the Delphi Method}

The Purposive Selection of Respondents: Delphi involves purposive selection of respondents; no standard social science sampling procedures exists. As Goldschmidt (1996) stated, the goal of purposive selection is to identify as many relevant viewpoints as possible, in the attempt to ensure that all relevant issues are identified and explored. The purposive selection has a serious impact on the outcome of the survey. The selection of the experts was through conference presentation on $\mathrm{H} \& \mathrm{~S}$, journal articles on $\mathrm{H} \& \mathrm{~S}$, personal contacts and practitioners dealing with H\&S. Their conduct details were through e-mails. This contact was obtained in order to forester communication. The H\&S experts were informed of the essence of the study in the introductory questionnaire survey. In order to qualify as an expert the following had to be fulfilled, each individual was required to meet at least three of the following minimum requirements: 1) minimum five years of work experience in either academia or industry; 2) at least one professional qualification: 3) an editor, book, chapter authorship: 4) minimum qualification for industry practitioners diploma and academics bachelor degree: 5) five or more publications in conferences and journals: 6) member or committee chair of faculty, 7) safety association member and 8) offers workshop or training in H\&S. The H\&S experts had to fulfill at least three of the eight requirements. A previous study by Rodgers and Lopez (2002) required the experts to attain at least two requirements out of five.

Optimal sample size of respondents using Delphi technique has not been established. However, literature has published research based on samples that vary from 10 and 50 as indicated by Campbell and Cantrill (2001). Furthermore, literature on use of Delphi method has supported a homogenous group of experts. Hence good results can be obtained with small panels of 10-15 individuals (Ziglio, 1996). Furthermore, recent study of Rajendran and Gambatese (2009) used a panel of 12 experts. Goldschmidt (1996) suggested that a 66\% response rate is adequate and Van Beek (1996) planned for a 75\% response rate for his Delphi survey; however $66.67 \%$ of the experts contacted agreed to participate in his research project. In the current study a total of 30 experts of H\&S were identified of which 20 agreed to participate after completing the introductory questionnaire survey. This was deemed to be an effective and efficient method as indicated by Okoli and Pawlowski, (2004). The response rate was $66.67 \%$, which was considered to be adequate as supported by Goldschmidt (1996) and Van Beek (1996). A few experts who did not consent to participate gave reasons such as:

"The Delphi method is an onerous task"

"I am currently busy hence will not be able to take part in this survey"

"I am currently busy and I have three children to take care of"

The experts resided in different parts of the world. This is because the validated leading indicators can be used in other developing countries and the developed countries to improve H\&S performance in projects undertaken by construction SMEs. Especially with the on-going debate of what should constitute the H\&S indicators for measuring performance at project level and organization level (Lingard and Rawlinson, 2005). The experts were from Australia (6), America (1), South Africa (7), Italy (1), Portugal (2), Ireland (1), Scotland (1), and Pakistan (1). The majority of experts from UK and America who were invited to participate declined the opportunity in writing or did not respond. The panel consisted of academics and industry practitioners.

Carrying out the Survey: Addler and Sainsbury (1996) suggested that Delphi survey should consist of three to four rounds that evolve from a loose and unstructured question to a more precise and structured exploration of the important issues. Hsu and Sandford (2007) on the other hand suggested that in order to improve on the response rate a structured questionnaire can be used in the first round especially where information on the subject matter is available. This study used the latter approach in order to improve on the response of the experts as indicated by Hsu and Sandford (2007) and to avoid Delphi fatigue which can be experienced by the experts as indicated by Drodge (1983) and Linstone and Turoff (1975). A well designed 
four round survey was used to validate and identify critical indicators of H\&S that will be used by SMEs in South Africa to measure and improve there H\&S performance at project level.

Preparing the Respondents for the Survey: An essential part of conducting any research is explaining to the respondents the purpose of the research and the intended outcomes. If respondents do not understand the aim of the Delphi exercise, they may answer the questions inappropriately or become frustrated and lose interest (Ziglio, 1996). When initial contact was made with the respondents the purpose of the research was explained clearly and concisely. This ensured the respondents knew the level of the research and the direction it was taking. The experts were selected before completing the questionnaire sent to them. Furthermore, the researchers assumed that the H\&S experts would naturally be happy to contribute to the research discourse in their field of expertise. Any researcher who thinks like this is taking a big risk, especially when his respondents are university professors and industry practitioners. Linstone and Turoff, (1975) indicated that a Delphi survey should provide the atmosphere of a fruitful communication process among peers. A well managed Delphi survey should be a highly motivating (Ziglio, 1996) task for the experts to be involved in, and this was the intension of the researcher. The Delphi survey was to take four months but it ended up taking 10 months due to an additional round after the third round was completed in January 2011. An additional round of Delphi was conducted which was advocated by the co-author to solidify the respondents response in each indicator. The fourth round took place between April 2011 and June 2011.

Methods of Reaching Consensus: It has been indicated that consensus forming is the essence of the Delphi technique. It can be defined as a gathering around median responses with minimal divergence (Murray \& Hammons, 1995). The researcher should carefully determine in advance what particular definition of consensus is to be used in his/her study. Critics of the Delphi find the issue of consensus one of the most contentious components of the method (Crisp et al., 1997). The building of consensus using various parameters was decided upon, the parameters to derive consensus was based on both the importance and the impact scale. The two scales had to complement each other. The median importance ratings of 9 to 10 and rating of $50 \%$ and above were deemed to attain consensus. The impact percentage median rate was $90 \%$ to $100 \%$ with a participant rating of $50 \%$ and above. However, other ways of defining consensus is the acceptance of ratings higher than a previously determined number by at least $51 \%$ of the participants and the elimination of topics that are vigorously opposed (Fink et al., 1984).

In the successive rounds no indicators were omitted apart from two, where the supervisor who is a health and safety specialist piloted the round 2 Delphi questionnaire. One statement was omitted and the other statement was merged after round 2, hence the reduction of the statements/indicators were now 62 in round 3 and 4 from the previous 64 . The essence of not omitting the indicators after the successive rounds as other researchers, (see Rajendran and Gambatese, 2009) was to check the consistency of the respondents and their stability. Resistance to consensus in the form of scattered distributions or outlying opinions should be considered carefully as they may yield new perspectives on the issues under investigation (Critcher and Goldstone, 1998). The indicators that never attained consensus were omitted at the end of the fourth round of the Delphi survey. The omission of the indicators was achieved based on the impact scale and importance scale. As previously indicated the results had to complement each other.

Pilot Study: The structured Delphi questionnaire survey was developed from extensive literature review hence was to be validated before it was sent to the experts. A pilot study was undertaken which included a member of the panel of experts and the supervisor who are experts in health and safety. The statistician from STATKON department at the University of Johannesburg statistics department verified the scale to be used and also clarified the wording of the statements/indicators. This approach was adapted from a study by Nichol (2007). This ensured the face validity of the questionnaire. The questionnaire for round 1 of Delphi was refined especially the wording of the statements to be more readable and easy to understand. The essence of not using all the H\&S experts to pilot the questionnaire was to eliminate any attrition after the first round of Delphi (see, Cape, 2004), which is a common trend (see Hsu and Sandford, 2007) when this approach is used in the first round.

Round 1 Success and Challenges: Lessons Learnt: The first round of Delphi survey has been termed the exploration phase (Ziglio, 1996), where respondents explore the question and add new material. The 
approach in the study conducted had a different approach as indicated by Hsu and Sandford (2007), where a structured questionnaire was used in the first round and new ideas were added in the fourth round. The approach is discussed across this article. In the first round of Delphi which commenced in September 2010 two questions were raised by two experts, based on the questionnaire and clarity of instructions in terms of competence. The explanations were replied to the experts individually. Some further questions that were asked by the experts were:

One expert asked "if the questionnaire was validated" and

Another expert asked if the "the response were to be based on the competence of the employee"

This is the essence of the Delphi approach as it is supposed to create discussion (see Ziglio, 1996). The questions were helpful and were clarified to the experts. In the first round of Delphi the experts were asked to rate the importance and impact of the indicators without adding any indicators. This approach of Delphi differs from the traditional Delphi which gave experts an opportunity to add any statements or indicators that they thought are vital and have been omitted for example in this study health and safety (H\&S) performance indicators or statements that will improvement H\&S in SMEs projects. The essence of the researchers not allowing the experts to add any indicators was based on the research objective and question developed in the first round. Where the experts were asked to rate the statements/indicators on a 10 point Likert scale of importance and impact. The other reason was to use a different approach as the researchers wanted to own the Delphi approach as mentioned previously (see Cape, 2004). A total of 20 questionnaires were sent to the experts who agreed to participate in this study. Past studies for example Hsu and Sandford (2007) indicated a tendency of attrition when using Delphi method, hence caution had to be taken and constant reminders had to be sent to the experts bearing in mind there busy schedule. In round one 13 experts responded promptly, where as the other six experts were sent reminders via email, Bertin (1996) stated that the care and attention with which the questionnaire is answered by the experts is a function of their degree of motivation and the time taken in replying is to a large extent a consequence of the factor of motivation. After round one 18 experts responded, of which the analysis of round one was administered by the researchers and the questionnaire for round two was prepared and sent to the experts. Two experts who did not respond in round one did not give reasons why they did not participate even after successive reminders. Finally those experts were excluded from this study. The impact of the withdrawal of the two experts was slight, as the other experts gave the required information.

Round 2 Success and Challenges: Lessons Learnt: In round 2 of Delphi method the experts were sent the feedback of round one with there rating highlighted in yellow and the group median inserted in a separate column of there round two Delphi questionnaire. Further questions were inserted in the Delphi questionnaire round 2 where experts were given options of changing there rates and conform to the group median, or if they do not want to change and they are falling out of the required scale they should give reasons why they have the differences. Being an iteration process the experts were to give reasons some experts gave reasons and others did not. In this round a few researchers responded late and they had to be reminded of the due date and the extension of the submission date. In round two of Delphi 18 experts responded of which the number was equivalent to those who responded in round 1 . The researchers who never changed there rating and were falling out of the group median within two unit Likert scale indicated that "SMEs do not have the expertise in using some of those indicators" or they "the SMEs do not have the competency and resources to undertake some actions stated". Two experts who changed there ratings in some of the statements indicated that they had made a mistake in there rating in round one. After round 2 one expert indicated the "difficulty of differentiating between the scale of importance and impact". The stability of respondents rating started to be evident in round 2 . The scale of impact indicated quite a number of statements with more than two units of the group median, in comparison with the importance scale.

Round 3 Success and Challenges: Lessons Learnt: In round 3 of Delphi method the experts were sent the feedback of round two with there rating highlighted in yellow and the group median inserted in a separate column of there round two Delphi questionnaire. The Delphi round 3 questionnaire was similar to round 2 experts were given options of changing there rating if they were two unit Likert scales point out of the group median, or if they do not want to change there rating they should give reasons. Being an iteration process the experts were to give reasons some experts gave reasons while others did not. The experts who never changed there rating and were falling out of the group median within two unit Likert scale on the 10 point Likert scale 
of importance and impact indicated that "SMEs do not have the expertise in using some of those indicators" or they "the SMEs do not have the competency and resources to undertake some actions stated". In this round a few experts responded late, they were sent reminders and the adjusted submission date. In round three of Delphi 16 experts responded, hence two experts were omitted, one expert responded late but the other had relocated to a different country and furnished the researcher with the new email address for further communication but did not respond. The scale of impact indicated quite a number of statements with more than two units out of the group median in comparison with the importance scale.

Round 4 Success and Challenges: Lessons Learnt: In round 4 of Delphi method the experts were sent the feedback of round three with their ratings highlighted in yellow and the group median inserted in a separate column of round four of Delphi questionnaire. This questionnaire in round four was similar to round 2 and 3 apart from a further additional question which required the experts to give any statement that they fill will improve H\&S performance at project level of SMEs. The experts were still given the options of changing there rates and conform to the group median, or if they do not want to change and they are falling out of the required scale they should give reasons. Being an iteration process the experts were to give reasons of there difference to the group median, some experts gave reasons and others did not. The experts who did not give reasons were sent a mail to indicate to them that they need to give reasons on the indicators that were not conforming to the group median. In this round a few researchers responded late and they had to be reminded of the due date and the extension of the submission date. In round four of Delphi 16 experts responded of which it was the same number of respondents as in round 3. Some of the experts added few indicators for example "the H\&S culture of SMEs has to change" and "clients have to be involved". In analyzing the proposed statements/indicators from the experts none of them were included in the final analyzed data. The scale of impact continued to indicate quite a number of statements with more than one outlier clustered around their group median in comparison with the importance scale. At the end of round 4 a total of 46 indicators were retained which indicated consensus. They were considered to be very important and had major impact in improving H\&S performance at project level of SMEs.

\section{Discussion}

Success of using the Delphi Method as an Inductive Approach: Conducting the Delphi survey was a rewarding experience and highly successful. In practice the Delphi method did prove to be well suited in validating and identifying the indicators and allowing the panel of experts to discuss without any interference from other experts. This finding concurs with other studies of (see Linstone \& Turoff, 1975; Ziglio, 1996). The respondents expressed there opinions and rated the indicators in a four round of Delphi survey. This Delphi approach was the appropriate method to gather opinions and initiate debate. The iterative nature of the Delphi method provided a structure within which important statements/indicators were validated and then discussed. The mode of communication, which was via email was viewed as a success, and concurs with Hsu and Sandford (2007) advocacy of using current technology. The use of a structured questionnaire and subsequent discussions in the successive rounds yielded success in this exploratory study as there was no high attrition rate of experts', four of the 20 experts were not able to participate in all the four rounds. This successful result can be inferred to the approaches discussed by Hsu and Sandford (2007), based on methods of reducing attrition in Delphi survey. The use of incentives which Hsu and Sandford (2007) also suggested was not used to entice the experts to respond. It is also important to mention that $93.75 \%$ that is 15 of the 16 experts who finished all the four rounds of Delphi gave comments on their ratings when they were two units above or below the group median as instructed in second, third and fourth round.

The experts were consistent in there comments and ratings, whereas a few of the experts agreed to change their rating marginally after the successive rounds. It cannot be verified if being out of the group median or the comments other H\&S experts had given and summarized in each round from round 2, 3 and 4 were a catalyst for change. However a few experts who changed there ratings indicated that they had made mistakes in their previous rounds when rating the statements/indicators. This indicates that the iterations in the various rounds yields concrete decisions unlike using a once off interview or survey in collecting data. The comments made by experts were quite motivating to indicate that this approach was a success. One expert indicated that "there should always be management commitment to improve health and safety practice". Another expert indicated that the "health and safety management system needs to be in place" 
Challenges of using the Delphi method: The summary of results of previous round of Delphi, were fed back to the respondents in appropriate time with the due date to return the questionnaire indicated, but not all respondents replied promptly. There was constant delay from a few respondents in all the successive rounds, this meant that the time scheduled for each round was extended by at least three weeks and hence infringes on the start of the round to follow. The third round took longer as is was in December some of the experts in South Africa were preparing for holidays hence could not respond to the questionnaire until January 2011, one expert in Australia was attending a conference in the United Kingdom, hence could not respond timely only until January 2011.

There was lapse of time between the third round and the fourth round of Delphi. This was caused by the coauthor of this paper advocating for the fourth round of Delphi in order to consolidate the consensus as some of the indicators had outliers in the impact scale. The experts had to be informed of the fourth round and they were sent the fourth round Delphi questionnaire with the results of the third round, with a further three questions. The fourth round survey was conducted from early April 2011 and ended in early June 2011. The challenge was to make sure the experts understood the importance of this additional round. Four of the experts withdrew from this research project, of which two experts did not give any reasons, one was late in the submission of the third round questionnaire and the fourth expert relocated to Malaysia from Australia. One expert did not comment on his ratings being out of the group median in all the successive rounds, even after being reminded. This expert had used Delphi method in his previous research work on health and safety, and was experienced in the use of this methodology.

Measures of Reaching Consensus: The use of different parameters to define consensus in this study was viewed as a success other than depending on one parameter. The use of median, rated importance between 9 to 10 and the impact rated between $90 \%$ to $100 \%$ and the percentage response rate of $50 \%$ and over between ratings of 9 to 10 indicating major impact and very important indicators to improve $\mathrm{H} \& \mathrm{~S}$ performance. This research project used all the mentioned parameters and ultimately eliminated the indicators that had no similar rating on the importance and impact. The elimination of the indicators, were therefore considered with great caution.

\section{Conclusion}

The criteria set for identifying the H\&S experts proved successful as out of the 30 experts invited to participate, the 20 experts who accepted to participate qualified as H\&S experts. The Delphi method also proved to be a success despite the challenges mentioned for example, the responding time of experts was poor. The success of reaching consensus using multiple parameters to decide on consensus is vital as only one or two parameters could be flawed and not giving the correct results. Furthermore the choice of the experts and the topic of discussion was a success, this is indicative of the attrition rate not being high, bearing in mind this research project adopted four successive rounds of Delphi, only 4 out of twenty experts did not complete all the four rounds, which to the researchers indicates that the approach used was a success and the topic was of interest to the majority of the experts. The researchers would also like to warn novice researchers to be careful when using this method. They should be careful with the approach they would like to adopt in the first round that is open ended or closed-ended questionnaire. The authors believe that these two different approaches could yield different response rate and findings of a particular study. The feedback process also needs to be taken into consideration and the type of questions to be asked in the successive rounds need to be decided before the commencement of the Delphi. The instructions of the questionnaire need to be clearly stated and be specific not ambiguous so as to achieve the correct results.

In order to improve on the response rate the authors are advocating for constant reminder to the experts shortly before the closing date and after the closing date of returning the questionnaire. Extension of time should be granted if the experts are not able to respond on the proposed date. The use of "polite" words such as please, thank you in advance etc. proved to be a success and the need for the researchers to be patient with the experts is deemed to be a success even if the experts did not respond after the first or second reminder these suggestion used in this present study, concur with the suggestions of Hsu and Sandford (2007). Despite the challenges and the lessons learnt when using Delphi method to identify and validate the H\&S indicators tailored for SMEs in construction industry in South Africa. It is worth noting that the Delphi approach was an 
appropriate method of great significance that identified the critical H\&S leading indicators for SMEs. These leading indicators are viewed as a channel that negates the popular use of lagging indicators e.g. accidents, injuries and death to measure H\&S performance in the construction industry. This study derives the importance of using Delphi method in the area of identifying leading indicators that are proactive. These indicators will inform construction SMEs of eventuality of an accident or injury to occur.

Possible Improvement to the Delphi Approach used in this Study: The main improvement deemed in this Delphi study is that as experts did not have face-to-face contact. There is a possibility that statements/leading indicators can be wrongly interpreted. Therefore, the experts could have been accorded the opportunity to comment on the statements. Furthermore, the need to use an open-ended questionnaire in the first round of Delphi could have assisted in thoroughly evaluating the experts' knowledge in this area of study.

Further Research: Based on the discussions in this paper the researchers are proposing the use of openended questionnaire in the first round of Delphi method, in a later study using the same team of experts. This will help in comparing the challenges and success of using an open ended questionnaire approach and the current closed-ended approach and also compare the final results of the two approaches.

\section{References}

Adler, M. \& Sainsbury, R. (1996), Alternative approaches to the computerization of social security: reflections on Delphi exercise: In Adler, M and Ziglio, E. (eds.) gazing into the oracle: the Delphi method and its application to social policy and public health, Jessica Kingsley Publishers.

Bertin, G. (1996). Delphi techniques and the planning of social services: the prevention of dependency among the old: In Adler, M and Ziglio, E. (eds.) Gazing into the oracle: the Delphi method and its application to social policy and public health, Jessica Kingsley Publishers.

Campbell, S. M. \& Cantrill, J. A. (2001), Consensus methods in prescribing research. Journal of Clinical Pharmacy and Therapeutics, 26(1), 5-14.

Cape, B. (2004). Gathering opinion and initiating debate: the success of the Delphi method in purely qualitative research. Library and Information, 28(89), 35-44.

Crisp, J., Pelletier, D., Duffield, C., Adams, A. \& Nagy, S. (1997). The Delphi method? Nursing Research, 46(2), 116-118.

Critcher, C. \& Goldstone, B. (1998). Utilizing the Delphi technique in policy discussion: a case study of a privatized utility in Britain. Public Administration, 76(3), 431-449.

Drodge, S. (1983). Delphi technique: Some practical considerations. Library and information research news, 6(21), 4-6.

Dyer, E. (1979). The Delphi technique in library research. Library research, 1(1), 41-52.

Fernandez-muniz, B., Montes-Peon, M. J. \& Vazquez-Ordas, J. C. (2007). Safety culture: Analysis of the causal relationships between its key dimensions. Journal of Safety Research, 38(6), 627-641.

Fink, A., Kosecoff, J., Chassin, M. \& Brook, R. H. (1984). Consensus methods characteristics and guidelines for use. American Journal of Public Health, 74(9), 979-983.

Goldschmidt, P. (1996). A comprehensive study of the ethical, legal and social implications of advances in biochemical and behavioral research and technology. In Adler, M and Ziglio, E. (eds.) Gazing into the oracle: the Delphi method and its application to social policy and public health, Jessica Kingsley Publishers.

Hallowell, M. R. (2008). A formal model for construction safety risk management. Unpublished doctoral dissertation, Oregon State University, U.S.A.

Hallowell, R. M. \& Gambatese, A. J. (2010). Qualitative research: application of the Delphi method to CEM research. Journal of Construction Engineering and Management, 136(1), 99-107.

Hsu, C. \& Sandford, A. B. (2007). Minimizing non-response in the Delphi process: How to respond to nonresponse, Practical assessment, research and evaluation, 12(17), 62-78.

James, J. M. \& Bolstein, R. (1992). Large monetary incentives and their effect on mail survey response rates. Public opinion quarterly, 56(4), 442-453.

Lingard, H. \& Rowlison, S. (2005). Occupational Health and Safety in Construction Project Management. Spon Press 2 Park Square, Milton Park Abington, Oxon OX 14 4rn. 
Linstone, H. \& Turoff, M. (1975). The Delphi method: Techniques and applications, Addison-Wesley publishing company.

Ludwig, B. (1994). Predicting the future have you considered using the Delphi methodology? Journal of Extension, 35(5), 1-4.

Murray, J. W. \& Hammons, J. O. (1995). Delphi: A Versatile Methodology for Conducting Qualitative Research. The Review of Higher Education, 18(4), 423-436.

Nichol, J. R. (2007). A framework for the development of Post-graduate Diploma programme in Mental Health. Unpublished PhD thesis, Faculty of Science, University of Free State, South Africa.

Okoli, C. \& Pawlowski, S. D. (2004). The Delphi method as a research tool: an example, design considerations and applications. Information and Management, 42(1), 15-29.

Rajendran, S. \& Gambatese, A. J. (2009). Development and initial validation of sustainable construction safety and health rating system. Journal of Construction Engineering and Management, 135(10), 10671075.

Rajendran, S. (2007), Sustainable Construction Safety and Health Rating System. Unpublished doctoral dissertation. Oregon State University, U.S.A

Rodgers, M. R. \& Lopez, E. C. (2002). Identifying critical cross-cultural school psychology competencies. Journal of School Psychology, 40(2), 115-141.

Van Beek, E. (1996). The use of Delphi method in forecasting accidents in the year 2000. In Adler, M and Ziglio, E. (eds.) Gazing into the oracle: the Delphi method and its application to social policy and public health, Jessica Kingsley Publishers.

Ziglio, E. (1996). The Delphi method and its contribution to decision making. In Adler, M and Ziglio, E. (eds.) Gazing into the oracle: the Delphi method and its application to social policy and public health, Jessica Kingsley Publishers. 